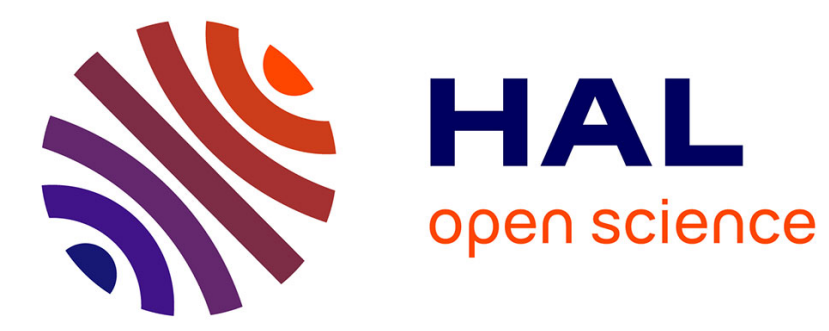

\title{
Taking caster wheel behavior into account in the kinematics of powered wheelchairs
}

Aline Baudry, Sylvain Guegan, Marie Babel

\section{To cite this version:}

Aline Baudry, Sylvain Guegan, Marie Babel. Taking caster wheel behavior into account in the kinematics of powered wheelchairs. Modelling, measurement and control C, 2018, 79 (4), pp.168-172. 10.18280/mmc_c.790403. hal-02124781

\section{HAL Id: hal-02124781 \\ https://hal.inria.fr/hal-02124781}

Submitted on 9 May 2019

HAL is a multi-disciplinary open access archive for the deposit and dissemination of scientific research documents, whether they are published or not. The documents may come from teaching and research institutions in France or abroad, or from public or private research centers.
L'archive ouverte pluridisciplinaire HAL, est destinée au dépôt et à la diffusion de documents scientifiques de niveau recherche, publiés ou non, émanant des établissements d'enseignement et de recherche français ou étrangers, des laboratoires publics ou privés. 


\section{Taking caster wheel behavior into account in the kinematics of powered wheelchairs}

Aline Baudry $^{1 *}$, Sylvain Guégan ${ }^{2}$, Marie Babel ${ }^{1}$

${ }^{1}$ Univ Rennes, INSA, CNRS, Inria, IRISA - UMR 6074, F-35000 Rennes, France

${ }^{2}$ Univ Rennes, INSA, LGCGM - EA 3913, F-35000 Rennes, France

Corresponding Author Email: aline.baudry@irisa.fr

https://doi.org/10.18280/mmc_c.790403

Received: 2 September 2018

Accepted:. 31 October 2018

Keywords:

smart wheelchair, caster wheels, kinematics, identification, driving

\begin{abstract}
The driving experience of an electric powered wheelchair (EPW) can be disturbed by unpleasant dynamic and kinematic effects of the caster wheels, particularly during maneuvers in narrow rooms and direction changes. In order to minimize this nasty behavior, we propose in this article a kinematic model of the wheelchair taking into account the effects of the caster wheels. The orientation of the caster wheels has been measured for different configurations: initial orientation, wheelchair velocity and user mass. The repeatability of the motions has been studied, and from these tests, their behavior has been modelled. This model has been then used to determine the wheelchair trajectory by using a more realistic kinematic. We have thus obtained a model allowing us to calculate more precisely the spaces that can be reach by powered wheelchairs, which are useful information to enhance control laws of their driving assistances.
\end{abstract}

\section{INTRODUCTION}

For the personal and cognitive development, it is important to explore the environment and to have social activities. However, this can be very difficult for people with reduced mobility. The use of a powered wheelchair can help them to be independent when they are unable to propel a manual wheelchair and when they need a third party for the most common tasks [1]. However, some of them cannot obtain the permission to drive an electric wheelchair because of visual and/or cognitive impairments. It has been shown that a large proportion of wheelchair users would benefit from using a smart wheelchair [2]. For that reason, since the early eighties, research works have been conducted to design smart wheelchairs and to develop autonomous and collision avoidance assisted wheelchairs [3-4].

Wheelchairs are usually modeled as a $(2,0)$ mobile robot [5]. The non-holonomic control problem induced from this model [6] can be avoided with sensor-based approaches [7], but the caster wheel kinematics is generally not taken into account. Yet the kinematic and dynamic effects of these wheels can be disturbing while driving. In particular, they can cause deviations from the desired trajectory during maneuvers and abrupt directions changes [8-9].

Hence, to assist the user to quickly and comfortably reach a location, it is important to take into account the caster wheel effect in the wheelchair dynamic behavior. These passively orientable wheels are designed to follow the wheelchair motion along non-straight paths. Their kinematic behavior has been studied in [10] for manual wheelchairs for only smooth motions, when they follow the wheelchair motion. A dynamic model estimator has been then implemented on an ergometer to simulate straight and curvilinear paths of a manual wheelchair [11]. It has also been exploited in the development of assistances applied to manual wheelchairs [12] but it has never been used on powered wheelchairs

In addition, caster wheel dynamical effects induced by direction changes have not been modelled, whereas collisions mostly occurred in such situations [13]. Hence, designing a maneuver assistive control law requires the definition of a related model. The dynamic behavior of the caster wheels depends on their frictions with the ground, and on the rolling resistance which oppose to their self-orientation motion [14]. If the Pacejka's magic formula [15] provides a complete model of any wheel behavior, it requires a good knowledge about the nature of the ground. Nevertheless, to implement an assistive control-law taking into account the caster wheel behavior, it is not feasible to consider the multiple grounds encountered during the navigation because of the complexity to characterize their properties in real-time. In [16], a compensation scheme based on odometry measurement prevents the wheelchair to drift because of misaligned caster wheels. This assistance is mostly based on odometry and, for a low-cost and pragmatic assistance solution, we aim to develop a sensorless control law. For such a scheme, a behavior model is required.

This paper is organized as follows. First, section II presents the wheelchair kinematics and the caster wheels kinematics. The caster wheels' behavior during direction changes is defined in section III and applied in section IV to compute the wheelchair kinematics. Finally, section V discusses the prospects of a control law which could be used for maneuvering assistance of powered wheelchairs.

\section{KINEMATIC MODELS OF THE WHEELCHAIR AND THE CASTER WHEELS}

Powered wheelchairs are modeled as a $(2,0)$ mobile non holonomic robot [5]. The kinematics resulting from the differential motorization of the wheelchair allows the driver 
to move along the longitudinal direction $(V)$ and to rotate $(\Omega)$ around z-axis. Thus two degrees of mobility make it possible to control the position and the orientation of the wheelchair.

Wheelchairs are equipped with caster wheels (from 2 up to 4 , depending on the configuration) that clearly influence the trajectory. Indeed, contrary to fixed wheels (Fig. 1), passive caster wheels or off-centered orientable wheels mounted onto wheelchairs present an offset $d$ (Fig. 2) which allows their self-orientation. If these wheels present the advantage of following non-linear trajectories, during abrupt changes of directions (a backward motion after a forward motion for example), they are opposed to the movement of the wheelchair before self-orientation. Then, the desired kinematics of the wheelchair is not respected, and trajectory deviations as well as brutal accelerations are observed. This transition motion is followed by a smooth trajectory, where caster wheels are aligned with the trajectory induced by differential wheels. We aim then at modelling this transition state that corresponds to a sudden reorientation of a caster wheel.

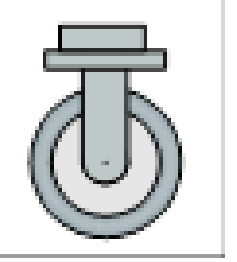

Figure 1. Fixed wheel

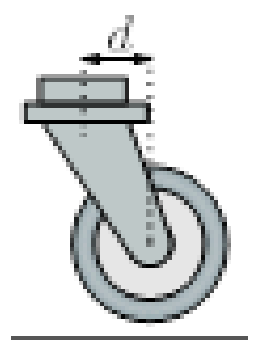

Figure 2. Passively steerable caster wheel

\subsection{Caster wheel velocity - steady state case}

Let $F_{W}(F, X, Y, Z)$ be the frame attached to the wheelchair. Let $F_{A_{i}}\left(A_{i}, x_{\mathrm{i}}, y_{\mathrm{i}}, z_{\mathrm{i}}\right)$ and $F_{C_{i}}\left(C_{i}, x_{\mathrm{i}}, y_{\mathrm{i}}, z_{\mathrm{i}}\right)$ be respectively the frames located on the steering axis $A_{i}$ of the caster wheel $i$ and the related ground contact point $C_{i}$ (Fig. 3). Let $V$ and $\Omega$ be the linear and angular velocities of the wheelchair. The velocity of the caster wheels is obtained at the ground contact point $\mathrm{C}$ with respect to frame, and depends on the velocity of the wheelchair itself. The steady state longitudinal velocity $\vec{V}_{\infty}$ the caster wheel is given by

$\vec{V}_{\infty}=\vec{V}+\overrightarrow{A_{l} F} \times \vec{\Omega}$

By denoting $\left(P_{X}, P_{Y}, 0\right)$ the position of the steering axis of the caster wheel, we obtain

$\overrightarrow{V_{\infty}}=\left(\begin{array}{c}V-P_{Y} \Omega \\ P_{X} \Omega \\ 0\end{array}\right)$
From this longitudinal velocity observed in steady state motion, we can calculate the angle $\alpha_{\infty}=\operatorname{atan} 2\left(P_{x} \Omega, V-\right.$ $\left.P_{y} \Omega\right)$ that the caster wheel should respect (Fig. 4).

\subsection{Caster wheel velocity - transient case}

During the transient state, the wheel velocity $\vec{V}_{i}$ is decomposed into a longitudinal velocity and a lateral velocity due to the angle $\left(\alpha_{\infty}-\alpha(t)\right)$ (Fig. 4). In the frame $R_{C_{i}}$, they are defined as

$\vec{V}_{\text {long } / R_{C}}=\left\|\vec{V}_{\infty}\right\| \times \cos \left(\alpha_{\infty}-\alpha(t)\right) \cdot \vec{x}$

$\vec{V}_{\text {lat } / R_{C}}=\left\|\vec{V}_{\infty}\right\| \times \sin \left(\alpha_{\infty}-\alpha(t)\right) \cdot \vec{y}$.

In the frame of the wheelchair $F_{W}$, we then calculate the longitudinal velocity as

$\vec{V}_{\text {long } / R_{F}}=\left(\begin{array}{c}\left\|\vec{V}_{\infty}\right\| \times \cos \left(\alpha_{\infty}-\alpha(t)\right) \cos (\alpha(t)) \\ \left\|\vec{V}_{\infty}\right\| \times \cos \left(\alpha_{\infty}-\alpha(t)\right) \sin (\alpha(t)) \\ 0\end{array}\right)$.

\subsection{Wheelchair kinematics along with caster wheel effects}

The kinematics of the wheelchair $(V(t), \Omega(t))$ can be determined from the combinations of the longitudinal velocity of each caster wheel, expressed in the wheelchair frame, so as

$$
\begin{aligned}
& V(t)=\frac{1}{n} \sum_{i=1}^{n} \vec{V}_{\operatorname{long}_{i} / R_{F}}(t) \cdot \vec{x}, \\
& \Omega(t)=\frac{1}{n} \sum_{i=1}^{n} \vec{V}_{\operatorname{long}_{i} / R_{F}}(t) \times \overrightarrow{A_{\mathrm{i}} F} \cos \left(\beta-\frac{\pi}{2}\right)
\end{aligned}
$$

where $\beta$ is the angle between $\vec{V}_{\text {long }_{i} / R_{F}}(t) \beta$ (Fig. 5) and $\cos \beta=\frac{\overrightarrow{A_{\mathrm{i}} F} \cdot \vec{V}_{\text {long }_{i} / R_{F}}(t)}{\left\|\overrightarrow{A_{\mathrm{i}} F}\right\| \times\left\|\vec{V}_{\text {long }_{i} / R_{F}}(t)\right\|}$

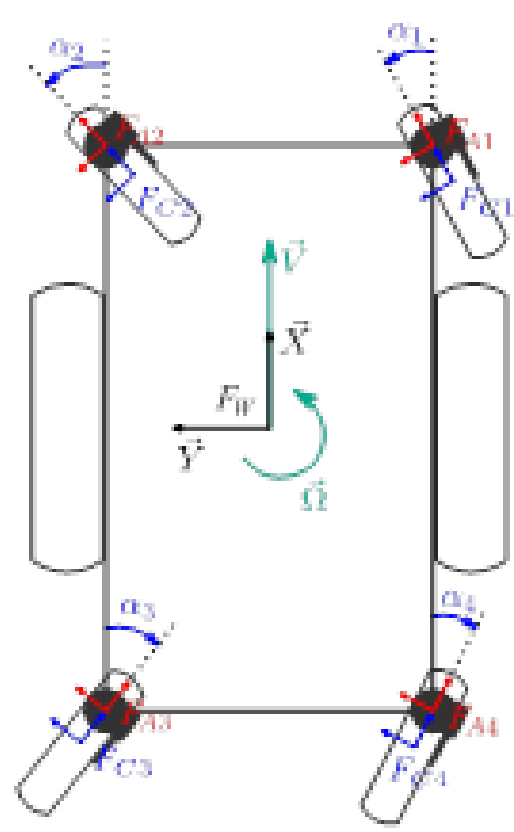

Figure 3. $F_{W}, F_{A_{i}}$ and $F_{C_{i}}$ frames 


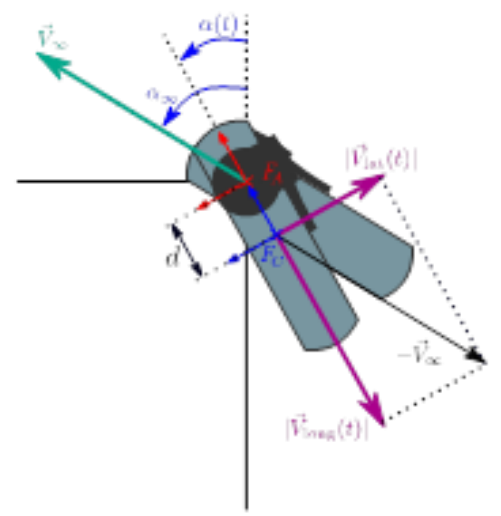

Figure 4. Kinematic relations in the front right caster wheel case

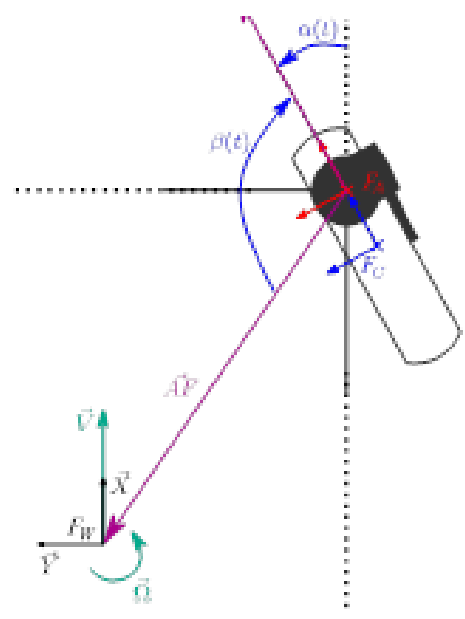

Figure 5. Caster wheel parameters

\section{CASTER WHEEL BEHAVIOR DURING DIRECTION CHANGES}

From the previous section, it has to be noted that the lateral velocity of the wheel will create a rotational movement because of the offset $d$, thus inducing a reorientation of the wheel. A kinematic study is thus not enough at this level since we must take into account the efforts that apply at the wheel. However, as we do not want to take into account the contact properties with the ground because of the complexity of the related identification, we propose to realize a kinematic study of the behavior. We conducted experimentations to obtain a transfer function realistic enough to use it in a control law.

\subsection{Data acquisition setup}

The characteristics of the sensors used for the instrumentation of the wheelchair (Fig. 6) are:

- Caster wheel orientation: Two incremental encoders with 1000 pulses per revolution, for a 0.36 degrees resolution, have been mounted on rotation axis of the caster wheels.

- Driving wheel angular velocity: Two incremental encoders with 12 pulses per revolution (1.23 degrees resolution) have been mounted on the motor axis.

- Wheelchair trajectory: Laser tracker Radian from API with a resolution of 0:08 $\mu \mathrm{m}$.
The data acquisition was realized by means of a Raspberry Pi 3 synchronized with the laser recording at the sampling rate $\mathrm{f}=100 \mathrm{~Hz}$. The control of the wheelchair was made thanks to a Tiva control card (Texas Instruments) connected to the CAN bus.

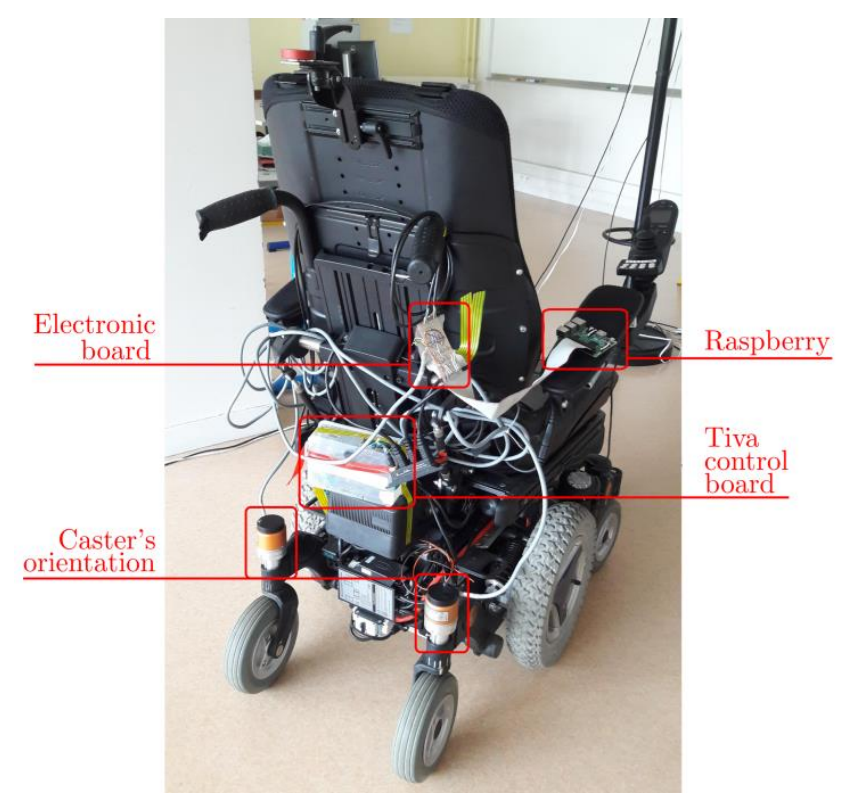

Figure 6. Instrumented wheelchair (Permobil M400) used for the experiment

\subsection{Experimental protocol}

In the design of this experiment, we choose to give the following values to the study parameters:

- Initial orientation of the caster wheels $\left(\alpha_{0}\right):\left(0^{\circ}, 30^{\circ}, 60^{\circ}\right.$, $\left.90^{\circ}, 180^{\circ}\right)$ as shown on Fig. 7

- Wheelchair velocity $(\mathrm{V}):(0.2,0.4,0.6)[\mathrm{m} / \mathrm{sec}]$;

- User mass $\left(\mathrm{M}_{\mathrm{R}}\right.$ relative mass): $(0,50)[\mathrm{Kg}]$.

During these experiments, we recorded the orientation of the caster wheels for 72 configurations of $\left(\alpha_{0}, V, M_{R}\right)$ while operating a forward-backward movement. For each configuration, 5 data sets were recorded.

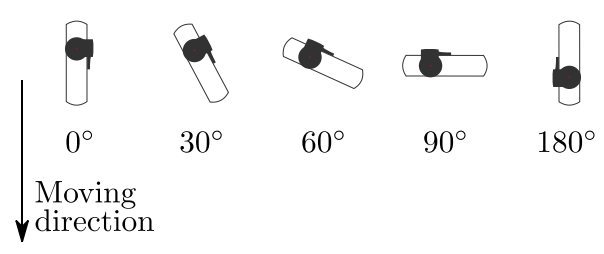

Figure 7. The different initial caster orientations used during the experiments

\subsection{Repeatability}

Before modeling the system, it is important to evaluate the repeatability of the observed behavior. This repeatability is verified if the correlation of the measurements for a given configuration is equal to 1 . Figure 8 highlights in particular the non-repeatability of the behavior for the $0^{\circ}$ initial orientation. It outlines that the caster wheels do not behave the same for the 5 successive experiments, which present different delays and rotation directions. The analysis of the trajectories leads to the same conclusion. 

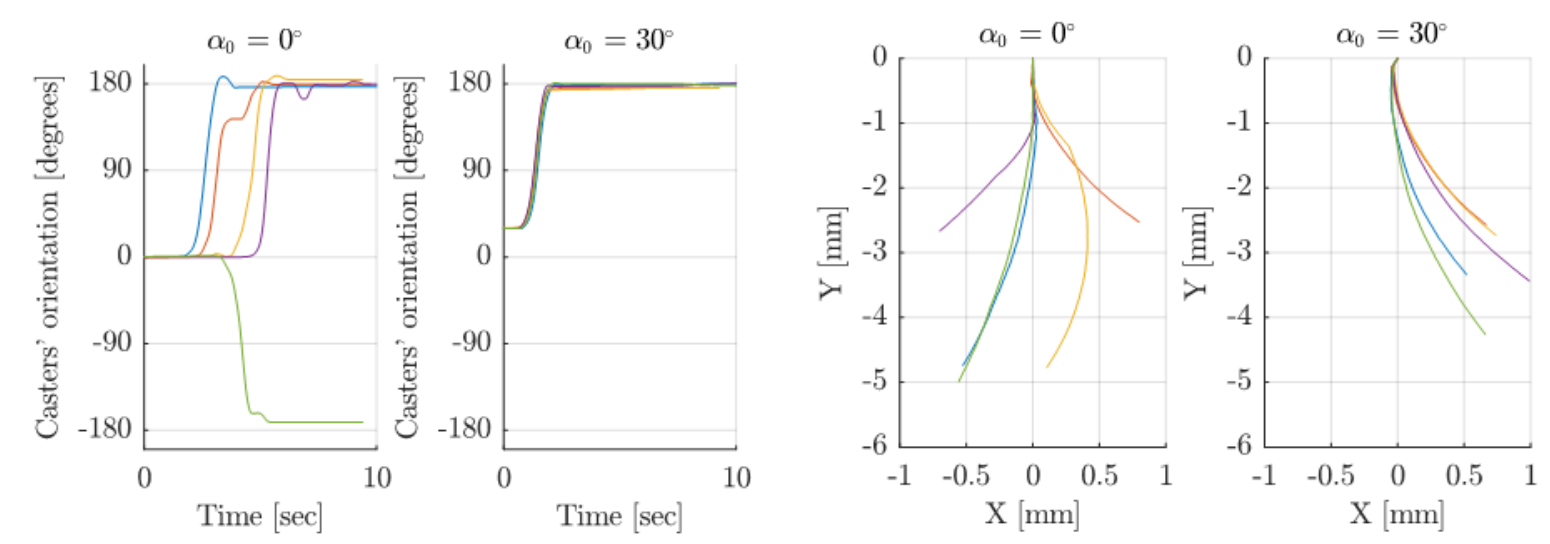

Figure 8. Rear caster wheel orientation evolutions and associated trajectories while going backward for $0.4 \mathrm{~m} / \mathrm{sec}$ velocity and 0 $\mathrm{Kg}$ user mass for different initial caster orientation $\left(\alpha_{0}\right)$

As our measurements are repeatable for the $30^{\circ}, 60^{\circ}$ and $90^{\circ}$ initial orientations, we can now model the caster wheel behavior.

\subsection{A transfer function to model the behavior}

We choose to model the caster wheels self-orientation with a transfer function to, afterwards, implement a dedicated control law. In a first step, we identify the $1^{\text {st }}, 2^{\text {nd }}$ and $3^{\text {rd }}$ order transfer functions corresponding to our measurements. The related transfer functions $H_{1} s t(s), H_{2}$ nd $(s)$ and $H_{3} r d(s)$ are defined as:

$H_{1} s t(s)=\frac{K}{1+\tau_{1} s} e^{-T_{0} s}$

$H_{2} n d(s)=\frac{K}{\left(1+\tau_{1} s\right)\left(1+\tau_{2} s\right)} e^{-T_{0} s}$

$H_{3} r d(s)=\frac{K}{\left(1+\tau_{1} s\right)\left(1+\tau_{2} s\right)\left(1+\tau_{3} s\right)} e^{-T_{0} s}$

where $K$ is a static gain, $T_{0}$ the time delay [sec], and $\tau_{1}, \tau_{2}, \tau_{3}$ the time constants [sec]. For the identification of the system, we use a trust-region-reflective algorithm on the function parameters $\left(K, T_{0}, \tau_{1}, \tau_{2}, \tau_{3}\right)$ and use the root-mean-square between the data and the computed model as the error term. This identification gave us very good results (correlation $>$ 93\%) for the three different transfer functions (Table 1). As the 1st, 2nd and 3rd order transfers functions give then similar results and the addition of a time constant does not greatly increase the correlation with the data, we decided to model the caster wheel behavior with a simple $1^{\text {st }}$ order transfer function.

Table 1. Correlation between the transfer functions and the data

\begin{tabular}{|c|c|}
\hline $\begin{array}{c}\text { Transfer } \\
\text { function }\end{array}$ & Correlation [\%] \\
\hline $1^{\text {st }}$ order & $93.680 \pm 1.1698$ \\
\hline $2^{\text {nd }}$ order & $95.362 \pm 1.1288$ \\
\hline $3^{\text {rd }}$ order & $95.957 \pm 1.1523$ \\
\hline
\end{tabular}

We then applied this $1^{\text {st }}$ order transfer function to the previously defined kinematic model and we observe a good correlation of our model with the ground truth (Fig. 9).

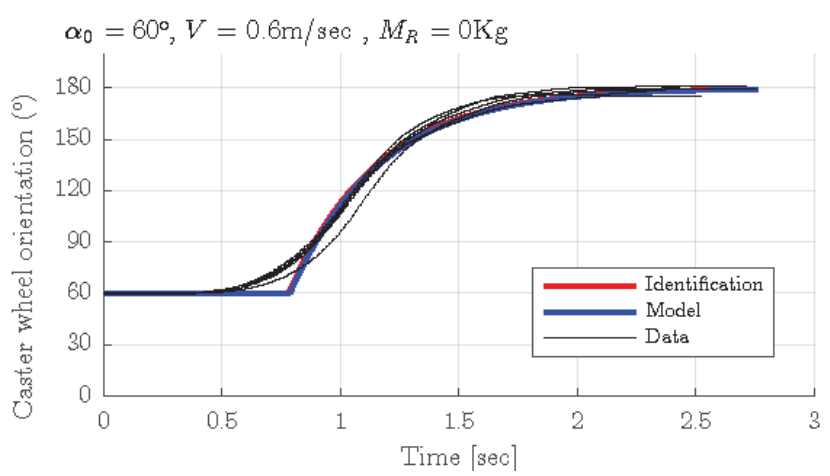

Figure 9. Modeled and identified transfer functions together with ground truth data

\section{DISCUSSION}

In this study, we designed a kinematic model of the wheelchair that integrated the caster wheels effects during changes of direction. The behavior of the caster wheels has been modeled with a 1st order transfer function which appears as a good tradeoff between accuracy and complexity. This model will be used to enhance driving assistances of powered wheelchair. The aim will be to minimize the selfalignment time of the caster wheels as well as the jolt felt during maneuvers.

In this study, we used only two encoders to measure the orientation of the rear caster wheels, assuming that the behavior of the two front caster wheels was similar. In future works, we will use four encoders to investigate if there is an influence of the rear caster wheels on the front caster wheels. Another statement is that the behavior obtained with the $0^{\circ}$ initial orientation of the caster wheels cannot be modeled because of the non-repeatability of the motion. Thus, relying on the space analysis [7], the motion has to be forced to initially orientate the caster wheels and then use the knowledge that we have on their behavior.

\section{CONCLUSION}

The objective of this paper was to model the behavior of the caster wheels of powered wheelchairs while direction changes occurred, to enhance maneuver assistance control laws. Results have shown that the 1 st order transfer function is sufficient enough to model the caster wheel behavior, 
leading to a simple parametric model. Thus, the real kinematics of the wheelchair with regard to the orientation of caster wheels could be computed, in order to perfectly control its motion.

In future works, we will focus on the design of a control law that assists the user during maneuvers and abrupt direction changes. In addition, we envisage to implement this model in a virtual reality powered wheelchair driving simulator [17], which takes into account the effects of the caster wheels.

\section{ACKNOWLEDGEMENTS}

This work is carried out as part of the INTERREG VA FMA ADAPT project "Assistive Devices for empowering disAbled People through robotic Technologies http://adaptproject. com/index.php.”. The Program is funded by the European Regional Development Fund (ERDF). Authors would also like to thank Eric Bazin, François Pasteau (INSA/IRISA), and Sylvain Rigaud.

\section{REFERENCES}

[1] Cooper RA. (1998). Wheelchair selection and configuration. Demos 2-3.

[2] Simpson RC, Lopresti EF, Cooper RA. (2008). How many people would benefit from a smart wheelchair? J. Rehabil. Res. Dev 45(1): 53-71. https://doi.org/10.1682/JRRD.2007.01.0015

[3] Simpson RC. (2005). Smart wheelchairs: A literature review. J. Rehabil. Res. Dev 42(4): 423-436. https://doi.org/10.1682/JRRD.2004.08.0101

[4] Ding D, Cooper RA. (2005). Electric powered wheelchair. IEEE Control Syst 25(2): 22-34.

[5] Campion G, Chung W. (2008). Wheeled robots. Springer Handbook of Robotics, pp. 391-410. https://doi.org/10.1007/978-3-540-30301-5_18

[6] Morin P, Samson C. (2008). Motion control of wheeled mobile robots. Springer Handbook of Robotics, pp. 799 826. https://doi.org/10.1007/978-3-540-30301-5_35

[7] Devigne L, Karakkat Narayanan V, Pasteau F, Babel M. (2016). Low complex sensor-based shared control for power wheelchair navigation. IEEE International Conference on Intelligent Robots and Systems. https://doi.org/10.1109/IROS.2016.7759799

[8] Ding D, Cooper RA, Guo S, Corfman TA. (2004). Analysis of driving backward in an electric-powered wheelchair. IEEE Trans. Control Syst. Technol 12(6): 934-943. https://doi.org/10.1109/TCST.2004.833638

[9] Guo S, Cooper RA, Corfman T, Ding D, Grindle G. (2003). Influence of wheelchair front caster wheel on reverse directional stability. Assist. Technol 15(2): 98104. https://doi.org/10.1080/10400435.2003.10131893

[10] Chenier F, Bigras P, Aissaoui R. (2011). An orientation estimator for the wheelchair's caster wheels. IEEE Trans. Control Syst. Technol 19(6): 1317-1326. https://doi.org/10.1109/TCST.2010.2084577

[11] Chénier F, Bigras P, Aissaoui R. (2011). A new dynamic model of the manual wheelchair for straight and curvilinear propulsion. IEEE International Conference on Rehabilitation Robotics 18(10): 10311043. https://doi.org/10.1109/ICORR.2011.5975357

[12] Xi L. (2016). Force sensorless power assist control for wheelchair on flat road using recursive least square with multiple forgetting. IEEE International Workshop on Sensing, Actuation and Motion Control, SAMCON.

[13] Gaal RP, Rebholtz N, Ralf H, Pfaelzer PF. (1997). Wheelchair rider injuries: Causes and consequences for wheelchair design and selection. J. Rehabil. Res. Dev 34: 58-71. https://doi.org/10.1300/J094v05n02_03

[14] Johnson BW, Aylor JH. (1985). Dynamic modeling of an electric wheelchair. IEEE Trans. Ind. Appl IA-21(5): 1284-1293. https://doi.org/10.1109/TIA.1985.349556

[15] Pacejka HB, Bakker E. (1992). The magic formula tyre model. Veh. Syst. Dyn 21: 1-18. http://dx.doi.org/10.1080/00423112-8969994

[16] Lee DA, Jung DG, Woo KS, Kim LK, Mok H, Han S. (2013). Orientation compensation for initially misaligned caster wheels. International Journal of Control, Automation and Systems 11(5): 1071-1074. https://doi.org/10.1007/s12555-012-9112-6

[17] Devigne L, Babel M, Nouviale F, Narayanan V, Pasteau F, Gallien P. (2017). Design of an immersive simulator for assisted power wheelchair driving. IEEE Int. Conf. Rehabil. Robot 17. https://doi.org/10.1109/ICORR.2017.8009379 